\title{
Social Networking Addiction: Emerging Themes and Issues
}

\section{Mark D Griffiths*}

International Gaming Research Unit, Psychology Division, Nottingham Trent University, UK

Social Networking Sites (SNSs) are virtual communities where users can create individual public profiles, interact with real-life friends, and meet other people based on shared interests [1]. SNS usage patterns from both consumer research and empirical research indicate that overall, regular SNS use has increased substantially over the last few years [1]. SNSs are predominantly used for social purposes, mostly related to the maintenance of established offline networks, relative to individual ones [1]. However, recent evidence suggests that individuals may feel compelled to maintain their online social networks in a way that may, in some circumstances, lead to using SNSs excessively.

In many areas of behavioral addiction, there has been debate about whether some excessive behaviors should even be considered as genuine addictions (e.g., video game playing, internet use, sex, exercise, etc.) and the same debate holds for addiction to social networking. Griffiths [2] has operationally defined addictive behavior as any behavior that features what he believes are the six core components of addiction (i.e., salience, mood modification, tolerance, withdrawal symptoms, conflict, and relapse). He also argues that any behavior (e.g., social networking) that fulfils these six criteria can be operationally defined as an addiction.

Researchers have suggested that the excessive use of new technologies (and especially online social networking) may be particularly problematic to young people [3]. In accordance with the biopsychosocial framework for the etiology of addictions [2], and the syndrome model of addiction [4], it is claimed that those people addicted to using SNSs experience symptoms similar to those experienced by individuals who suffer from addictions to substances or other behaviors [3]. This has significant implications for clinical practice because unlike other addictions, the goal of SNS addiction treatment cannot be total abstinence from using the internet per se it is an integral element of today's professional and leisure culture [1]. Instead, the ultimate therapy aim is controlled use of the internet and its respective functions, particularly social networking applications, and relapse prevention using strategies developed within cognitivebehavioral therapies [3].

To explain the formation of SNS addiction, Turel and Serenko [5] recently summarized three overarching theoretical perspectives that may not be mutually exclusive:

- Cognitive-behavioral model: This model emphasizes that 'abnormal' social networking arises from maladaptive cognitions and is amplified by various environmental factors, and eventually leads to compulsive and/or addictive social networking.

- Social skill model: This model emphasizes that 'abnormal' social networking arises because people lack self-presentational skills and prefer virtual communication to face-to-face interactions, and it eventually leads to compulsive and/or addictive use of social networking.

- Socio-cognitive model: This model emphasises that 'abnormal' social networking arises due to the expectation of positive outcomes, combined with internet self-efficacy and deficient internet self-regulation eventually leads to compulsive and/or addictive social networking behavior.
Based on these three models, $\mathrm{Xu}$ and Tan [6] suggest that the transition from normal to problematic social networking use occurs when social networking is viewed by the individual as an important (or even exclusive) mechanism to relieve stress, loneliness, or depression. They contend that those who frequently engage in social networking are poor at socializing in real life. For these people, social media use provides such people continuous rewards (e.g. self-efficacy, satisfaction) and they end up engaging in the activity more and more, eventually leading to many problems (e.g., ignoring real life relationships, work/ educational conflicts, etc.). The resulting problems may then exacerbate individuals' undesirable moods. This then leads such individuals to engage in the social networking behavior even more as a way of relieving dysphoric mood states. Consequently, when social network users repeat this cyclical pattern of relieving undesirable moods with social media use, the level of psychological dependency on social networking increases.

A behavioral addiction such as SNS addiction may thus be seen from a biopsychosocial perspective [2]. Just like substance-related addictions, it would appear that in some individuals, SNS addiction incorporates the experience of the 'classic' addiction symptoms, namely mood modification (i.e., engagement in SNSs leads to a favorable change in emotional states), salience (i.e., behavioral, cognitive, and emotional preoccupation with the SNS usage), tolerance (i.e., ever increasing use of SNSs over time), withdrawal symptoms (i.e., experiencing unpleasant physical and emotional symptoms when SNS use is restricted or stopped), conflict (i.e., interpersonal and intrapsychic problems ensue because of SNS usage), and relapse (i.e., addicts quickly revert back to their excessive SNS usage after an abstinence period).

It is generally accepted that a combination of biological, psychological and social factors contributes to the etiology of addictions $[2,4]$ that may also hold true for SNS addiction. From this it follows that SNS addiction shares a common underlying etiological framework with other substance-related and behavioral addictions. However, due to the fact that the engagement in SNSs is different in terms of the actual expression of (internet) addiction (i.e., pathological use of SNSs rather than other internet applications), the phenomenon may be worthy of individual consideration, particularly when considering the potentially detrimental effects of both substance-related and behavioral addictions on individuals who experience a variety of negative consequences because of their addiction.

Research into social networking addiction has been relatively

*Corresponding author: Mark D Griffiths, International Gaming Research Unit Psychology Division, Nottingham Trent University, UK, Burton Street, Nottingham, NG1 4BU, United Kingdom, Tel: 0115-8482401; E-mail: mark.griffiths@ntu.ac.uk

Received December 13, 2013; Accepted December 18, 2013; Published December 28, 2013

Citation: Griffiths MD (2013) Social Networking Addiction: Emerging Themes and Issues. J Addict Res Ther 4: e118. doi: 10.4172/2155-6105.1000e118

Copyright: ( 2013 Griffiths MD. This is an open-access article distributed under the terms of the Creative Commons Attribution License, which permits unrestricted use, distribution, and reproduction in any medium, provided the original author and source are credited. 
sparse. According to a recent review [7], the twenty or so empirical studies examining SNS addiction fall into one of four types: (i) selfperception studies of social networking addiction, (ii) studies of social networking addiction utilizing a social networking addiction scale, (iii) studies examining the relationship between social networking and other online addictions, and (iv) studies examining social networking addiction and interpersonal relationships. The review noted that all the studies suffered from a variety of methodological limitations. Many of the studies attempted to assess SNS addiction, but mere assessment of addiction tendencies does not suffice to demarcate real pathology. Most of the study samples were generally small, specific, self-selected, convenient, and skewed with regards to young adults and female gender. This may have led to the very high addiction prevalence rates (up to 34\%) reported in some studies as individuals from these sociodemographic groups are likely to be more heavy social networking users. Consequently, empirical studies need to ensure that they are assessing addiction rather than excessive use and/or preoccupation.

Griffiths [8] recently noted that for many researchers, Facebook addiction has become almost synonymous with social networking addiction. However, Facebook is just one of many websites where social networking can take place. Most of the scales that have been developed have specifically examined excessive Facebook use such as the Bergen Facebook Addiction Scale [9], the Facebook Addiction Scale [10], and the Facebook Intrusion Questionnaire [11], i.e., addiction to one particular commercial company's service (i.e., Facebook) rather than the whole activity itself (i.e., social networking). The real issue here concerns what people are actually addicted to and what the new Facebook addiction tools are measuring.

For instance, Facebook users can play games like Farmville [12], can gamble on games like poker [13], can watch videos and films, and can engage in activities such as swapping photos or constantly updating their profile and/or messaging friends on the minutiae of their life $[1,8]$. Therefore, 'Facebook addiction' is not synonymous with 'social networking addiction' - they are two fundamentally different things as Facebook has become a specific website where many different online activities can take place - and may serve different purposes to various users. What this suggests is that the field needs a psychometrically validated scale that specifically assesses 'social networking addiction' rather than Facebook use. In the aforementioned scales, social networking as an activity is not mentioned, therefore the scale does not differentiate between someone potentially addicted to Farmville or someone potentially addicted to constantly messaging Facebook friends.
Whether social networking addiction exists is debatable depending upon the definition of addiction used, but there is clearly emerging evidence that a minority of social network users experience addictionlike symptoms as a consequence of their excessive use [7]. Studies endorsing only a few potential addiction criteria are not sufficient for establishing clinically significant addiction status. Similarly, significant impairment and negative consequences that discriminate addiction from mere abuse have (to date) generally not been assessed in published studies. Thus, future studies have great potential in addressing the emergent phenomenon of SNS addiction by means of applying better methodological designs, including more representative samples, and using more reliable and valid addiction scales so that current gaps in empirical knowledge can be filled.

\section{References}

1. Kuss DJ, Griffiths MD (2011) Online social networking and addiction--a review of the psychological literature. Int J Environ Res Public Health 8: 3528-3552.

2. Griffiths MD (2005) A 'components' model of addiction within a biopsychosocial framework. J Subst Use 10: 191-197.

3. Echeburúa E, de Corral P (2010) [Addiction to new technologies and to online social networking in young people: A new challenge]. Adicciones 22: 91-95.

4. Shaffer HJ, LaPlante DA, LaBrie RA, Kidman RC, Donato AN, et al. (2004) Toward a syndrome model of addiction: multiple expressions, common etiology. Harv Rev Psychiatry 12: 367-374.

5. Turel O, Serenko A (2012) The benefits and dangers of enjoyment with socia networking websites. Eur J Inf Syst 21: 512-528.

6. Xu H, Tan BCY (2012) Why do I keep checking Facebook: Effects of message characteristics on the formation of social network services addiction.

7. Griffiths MD, Kuss DJ, Demetrovics Z (2014) Social networking addiction: An overview of preliminary findings. In: Rosenberg K, Feder L (Eds.), Behaviora Addictions: Criteria, Evidence and Treatment (pp.119-141). Elsevier: New York.

8. Griffiths MD (2012) Facebook addiction: Concerns, criticisms and recommendations. Psychol Rep 110: 518-520.

9. Andreassen CS, Torsheim T, Brunborg GS, Pallesen S (2011) Development of a Facebook Addiction Scale. Psychol Rep 110: 501-517.

10. Cam E, Isbulan O (2012) A new addiction for teacher candidates: Social networks. Turkish Online Journal of Educational Technology 11: 14-19.

11. Elphinston RA, Noller $P$ (2011) Time to face it! Facebook Intrusion and the implications for romantic jealousy and relationship satisfaction. Cyberpsychol Behav Soc Networking 14: 631-635.

12. Griffiths MD (2010) Gaming in social networking sites: A growing concern? World Online Gambling Law Report 9: 12-13.

13. Griffiths MD, Parke J (2010) Adolescent gambling on the internet: a review. Int J Adolesc Med Health 22: 59-75.

Submit your next manuscript and get advantages of OMICS Group submissions

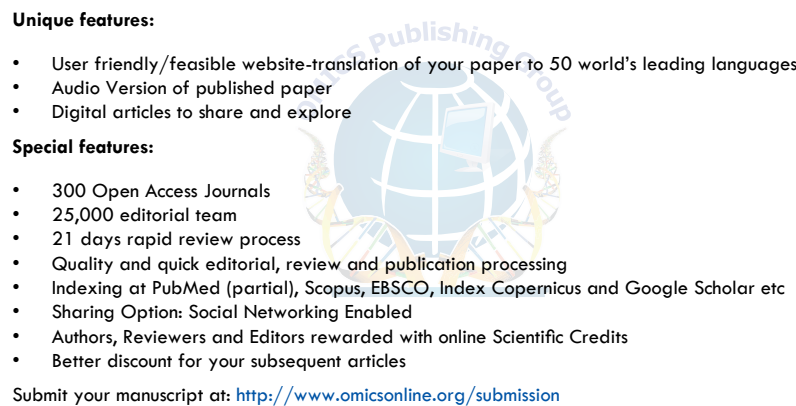

